\title{
Relatively mild symptoms after chronic intoxication with a double dose encorafenib: a case report
}

\author{
Paola Mian ${ }^{1}$, Elvira Meussen ${ }^{1}$, Djura Piersma $^{1}$, and Nienke Lankheet ${ }^{1}$ \\ ${ }^{1}$ Medisch Spectrum Twente
}

September 11, 2020

\begin{abstract}
Introduction: Encorafenib (BraftoviTM) is indicated for the treatment of adult patients with unresectable or metastatic melanoma with a BRAF V600 mutation, in combination with binimetinib (Mektovi (R)). According to the product label of encorafenib, there are no specific treatment recommendations in case of an overdose. Case presentation: We report on a 63 year old man who ingested a double dose $(900 \mathrm{mg})$ of encorafenib for 16 days. He developed overall minor chronic intoxication symptoms such as upset stomach, bloating, occasional vomiting and muscle pain. Based on the most occurring adverse events of encorafenib, liver values, kidney function parameters and QTc interval were measured. Kidney function parameters were normal, whereas liver values were slightly increased and QTc slightly prolonged. The plasma concentration 3 hours after the last dose was $2110 \mathrm{ng} / \mathrm{mL}$. Conclusion: We describe the course of a case with a chronic toxicity during 16 days of double dose of encorafenib as well as the followed approach, which could be taken into account when treating a patient with encorafenib intoxication.
\end{abstract}

\section{Introduction}

Encorafenib (Braftovi ${ }^{\mathrm{TM}}$ ) is indicated for the treatment of adult patients with unresectable or metastatic melanoma with a BRAF V600 mutation, in combination with binimetinib (Mektovi @) (1). Encorafenib, as a BRAF inhibitor, blocks the MAPK signaling pathway of the V600-mutated constitutively active BRAF molecule. Binimetinib, as a MEK inhibitor, blocks the downstream signaling pathway through the MEK molecule. In addition, MEK inhibitors reduce side effects by reduction of paradoxical activation of the MAPK pathway by BRAF inhibition (2). Approximately $40 \%$ of all metastatic melanoma tumors harbour an oncogenic BRAF V600 mutation (3). Encorafenib combined with binimetinib, slows or stops the enhanced cell growth and has shown to improve overall and progression-free survival among patients with metastatic melanoma (4). When combined, the recommended dose of encorafenib is $450 \mathrm{mg}$ once daily, and of binimetinib $45 \mathrm{mg}$ twice daily. The management of adverse reactions may require dose reduction, temporary interruption or treatment discontinuation of both or either one of the drugs (1). Although the product label provides recommendations in case of adverse events, there is no specific treatment recommendation in case of an overdose (1). We describe the course of a case with a chronic toxicity during 16 days of double dose of encorafenib as well as the followed approach, which could be taken into account when treating a patient with an encorafenib intoxication. 


\section{Case presentation}

In this case presentation we report on a 63 year old man treated for BRAF-mutated metastatic melanoma spread to lymph nodes, pleural cavity, lungs, liver as well as a suspected asymptomatic brain metastasis. Initially, the patient received dabrafenib in combination with trametinib resulting in excellent clinical and radiological response. Due to grade 2 pyrexia and chills the patient's treatment was discontinued and he was switched to ipilimumab in combination with nivolumab. After 2 courses he developed grade 3 colitis treated first with prednison and subsequently with infliximab. Unfortunately he showed progressive disease with new peritoneal metastases. In addition, he was in clinical distress caused by pleural effusion and ascites. He was re-challenged with dabrafenib plus trametinib, which again resulted in significant clinical and radiological response. However chills grade 2 also reoccurred and he was switched to encorafenib in combination with binimetinib, a regime known with low prevalence of pyrexia or chills as adverse events $(5,6)$. At that moment medication used by the patient was paracetamol $1000 \mathrm{mg}$ QID p.r.n., omeprazole $40 \mathrm{mg}$ BID, metoclopramide $10 \mathrm{mg}$ TID p.r.n; cotrimoxazol $480 \mathrm{mg}$ QD, fluconazole $100 \mathrm{mg}$ QD; salbutamol QID p.r.n; gastilox chewable tablet TID. Prednisolone dose was $10 \mathrm{mg}$ QD for 1 week, followed by $5 \mathrm{mg}$ or $10 \mathrm{mg}$ every other day, for the next week, followed by $5 \mathrm{mg}$ QD for a week, after which prednisolone intake stopped. For encorafenib the dose prescribed was $450 \mathrm{mg}$ QD (08.00h), consisting of 6 capsules of $75 \mathrm{mg}$ and $45 \mathrm{mg}$ BID (08.00h and 18.00h), consisting of 3 tablets of $15 \mathrm{mg}$ for binimetinib.

Sixteen days after having started the new oral oncolytic therapy the patient came to the hospital mentioning an upset stomach, bloating, occasional vomiting, and muscle pain probably caused by him working in and around the house. After an in depth anamneses of the patient it seemed that he had taken a double dose (900 mg QD instead of $450 \mathrm{mg}$ QD) encorafenib in combination with the prescribed dose of binimetinib (45 mg BID) for 16 days. After inquiring about the reason for taking this double dose, the patient confirmed that the nurse practitioner had discussed the medication prescription correctly with him, but he himself had not read the prescription with enough detail (flyer was too full with confusing pictograms). Additionally, due to the covid-19 pandemic, his medication was delivered at home, so he missed the additional information normally given at the first dispensing by the pharmacy. To treat the patient's chronic intoxication it was decided to opt for an observational approach, sustained by the fact that the product label did not mention any specific treatment in case of an overdose encorafenib and the patient did not mention suffering from any serious conditions besides the above-mentioned. Based on the most occurring adverse events of encorafenib, liver values, kidney function parameters and QTc interval were measured (Table 1). It can be concluded that kidney function was not affected by this chronic intoxication, whereas liver values (mainly LD, GGT) were slightly increased. The ECG showed a slightly prolonged QTc interval of $447 \mathrm{~ms}$ (reference value $<420 \mathrm{~ms}$ ).

Based on the pharmacokinetic profile of encorafenib such as short half-life $=6.32$ hours (1) which is independent of the dose and non-saturable absorption of encorafenib, no signs of accumulation and no delayed intoxication related symptoms were to be expected. However, because of lacking literature on encorafenib pharmacokinetics in case of a chronic intoxication it was decided to monitor plasma levels 3 hours after the last dose. Levels were measured at the Department of Pharmacy \& Pharmacology, Netherlands Cancer Institute, Amsterdam, the Netherlands, using a liquid chromatography-tandem mass spectrometric assay (7). This level turned out to be $2110 \mathrm{ng} / \mathrm{mL}$

It was decided to re-start the treatment with encorafenib in combination with binimetinib. As the patient had ingested a double dose of encorafenib for 16 days, he no longer had any encorafenib tablets available at home. As a new supply would be available after the weekend, it was decided to re-start encorafenib as well as binimetinib after 3 days after the last dose.

\section{Discussion}

Here we present a patient with a chronic toxicity following 16 days of ingestion of a double dose (900 $\mathrm{mg}$ ) of encorafenib. To the best of our knowledge, this is the first case report that described a detailed course of 
this intoxication, including the followed approach.

A prior report on encorafenib intoxications was mentioned in the product label (1). However, instead of a chronic intoxication it concerned an acute overdose. In 3 out of the 14 patients, kidney dysfunction (hypercreatinemia of grade 3) was observed after ingestion of 600 to $800 \mathrm{mg}$ encorafenib. The highest administered dose, as a result of a dosing mistake, occurred in a patient who ingested a dose of encorafenib of $600 \mathrm{mg}$ twice during one day (total dose of $1200 \mathrm{mg}$ ). The adverse events reported by this patient were events of nausea, vomiting and blurred vision grade 1, all disappearing afterwards. In general, the patient described in our case report showed also mild symptoms. Kidney function was not affected by this chronic intoxication, whereas liver values (mainly LD, GGT) were slightly increased. The ECG showed a slightly prolonged QTc interval. It has to be noted however, that increased LD and/or GGT does not have to be caused by the chronic intoxication, but may also be caused by the liver metastases. The mild symptoms occurring after the chronic intoxication also match with the plasma concentration level of $2110 \mathrm{ng} / \mathrm{mL} 3$ hours after the last intake. In literature information is available on maximum concentrations (Cmax) achieved after around 2 hours of administration of therapeutic encorafenib dosages. Delord et al. reported a median Cmax of $3800 \mathrm{ng} / \mathrm{mL}$ in 6 patients with long-term use (15 days) of encorafenib $450 \mathrm{mg}$ (8), while Sparidanset al. a Cmax of 1527 $\mathrm{ng} / \mathrm{mL}$ in one patient who ingested $200 \mathrm{mg}$ encorafenib (7). As encorafenib exhibits linear pharmacokinetics the above-mentioned Cmax can be extrapolated to an expected Cmax of $3436 \mathrm{ng} / \mathrm{mL}$ after administration of $450 \mathrm{mg}$. There seems to be no accumulation of encorafenib within the body as the accumulation ratio is 0.63 and with long-term use of encorafenib 0.45 (due to auto induction). Furthermore encorafenib has a short half-life, causing this Cmax reference value levels described in literature after one daily dosing to applied as a reference values in this case report. Extrapolating the plasma concentration level of $2110 \mathrm{ng} / \mathrm{mL}$ results in a Cmax (at 2 hours) of $2354.6 \mathrm{ng} / \mathrm{mL}$ being inline and far from increased in comparison with the reference concentrations reported in literature.

In conclusion, the fact that during long-term exposure to a double dose of encorafenib, both adverse events and plasma concentration level were acceptable, treatment can consist of closely monitoring the most important lab parameters (kidney, liver, QTc). This information may be useful for further treatment of intoxications with encorafenib.

\section{Table 1: Chemical profile during hospitalization}

\begin{tabular}{|c|c|}
\hline Lab parameters [unit], (reference value) & Value \\
\hline $\mathrm{Hb}[\mathrm{mmol} / \mathrm{L}],(8.5-11.0)$ & 7.6 \\
\hline Thrombocytes $\left[* 10^{9} / \mathrm{L}\right],(150-400)$ & 168 \\
\hline Leukocytes $\left[{ }^{*} 10^{9} / \mathrm{L}\right],(4-10)$ & 3.9 \\
\hline Urea, [mmol/L], (2.9-7.5) & 4.7 \\
\hline 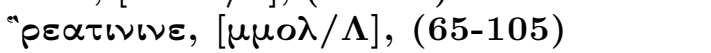 & 91 \\
\hline CKD-epi, $\left[\mathrm{ml} / \mathrm{min} / 1,73 \mathrm{~m}^{2}\right],(>64)$ & 77 \\
\hline Potassium, $[\mathrm{mmol} / \mathrm{L}],(3.5-5.0)$ & 4.3 \\
\hline Sodium, $[\mathrm{mmol} / \mathrm{L}],(135-145)$ & 134 \\
\hline $\mathrm{AF}[\mathrm{U} / \mathrm{L}],(20-135)$ & 77 \\
\hline ASAT $[\mathbf{U} / \mathbf{L}],(<40)$ & 19 \\
\hline ALAT $[\mathbf{U} / \mathbf{L}],(<45)$ & 11 \\
\hline $\mathbf{L D}[\mathbf{U} / \mathbf{L}],(<\mathbf{2 5 0})$ & 253 \\
\hline GGT $[\mathbf{U} / \mathbf{L}],(<50)$ & 93 \\
\hline Amylase $[\mathbf{U} / \mathbf{L}],(<\mathbf{1 0 0})$ & 43 \\
\hline Lipase $[\mathbf{U} / \mathbf{L}],(<60)$ & 35 \\
\hline
\end{tabular}

The bold printed values indicate the deviation relative to the reference. Abbreviations: AF: Alkaline phosphatase, ALAT: Alanine Aminotransferase, ASAT: Aspartate Aminotransferase, CKD-epi: Chronic Kidney Disease Epidemiology: GGT: Gamma-glutamyl transferase, Hb: Hemoglobin 


\section{References}

1. SmPC. Braftovi $50 \mathrm{mg}$ hard capsules 2020 [updated 06-11-2020. Available from: https://www.medicines.org.uk/emc/product/9499/smpc.

2. Belden S, Flaherty KT. MEK and RAF inhibitors for BRAF-mutated cancers. Expert reviews in molecular medicine. 2012;14:e17.

3. Rose AAN. Encorafenib and binimetinib for the treatment of BRAF V600E/K-mutated melanoma. Drugs of today (Barcelona, Spain : 1998). 2019;55(4):247-64.

4. Dummer R, Ascierto PA, Gogas HJ, Arance A, Mandala M, Liszkay G, et al. Encorafenib plus binimetinib versus vemurafenib or encorafenib in patients with BRAF-mutant melanoma (COLUMBUS): a multicentre, open-label, randomised phase 3 trial. Lancet Oncol. 2018;19(5):603-15.

5. Hamid O, Cowey CL, Offner M, Faries M, Carvajal RD. Efficacy, Safety, and Tolerability of Approved Combination BRAF and MEK Inhibitor Regimens for BRAF-Mutant Melanoma. Cancers. 2019;11(11).

6. Gogas HJ, Flaherty KT, Dummer R, Ascierto PA, Arance A, Mandala M, et al. Adverse events associated with encorafenib plus binimetinib in the COLUMBUS study: incidence, course and management. Eur $\mathrm{J}$ Cancer. 2019;119:97-106.

7. Sparidans RW, Rosing H, Rood JJM, Schellens JHM, Beijnen JH. Liquid chromatography-tandem mass spectrometric assay for therapeutic drug monitoring of the B-Raf inhibitor encorafenib, the EGFR inhibitors afatinib, erlotinib and gefitinib and the O-desmethyl metabolites of erlotinib and gefitinib in human plasma. Journal of chromatography B, Analytical technologies in the biomedical and life sciences. 2016;1033-1034:390-8.

8. Delord JP, Robert C, Nyakas M, McArthur GA, Kudchakar R, Mahipal A, et al. Phase I DoseEscalation and -Expansion Study of the BRAF Inhibitor Encorafenib (LGX818) in Metastatic BRAF-Mutant Melanoma. Clin Cancer Res. 2017;23(18):5339-48. 